Old Dominion University

ODU Digital Commons

Biological Sciences Faculty Publications

Biological Sciences

2003

\title{
Enhancement of Disease and Pathology by Synergy of Trichuris Suis and Campylobacter Jejuni in the Colon of Immunologically Naive Swine
}

\author{
Linda S. Mansfield \\ David T. Gauthier \\ Old Dominion University, dgauthie@odu.edu
}

Sheila R. Abner

Kathryn M.Jones

Stacey R. Wilder

See next page for additional authors

Follow this and additional works at: https://digitalcommons.odu.edu/biology_fac_pubs

Part of the Environmental Health Commons, Environmental Public Health Commons, and the Tropical Medicine Commons

\section{Repository Citation}

Mansfield, Linda S.; Gauthier, David T.; Abner, Sheila R.; Jones, Kathryn M.; Wilder, Stacey R.; and Urban, Joseph F., "Enhancement of Disease and Pathology by Synergy of Trichuris Suis and Campylobacter Jejuni in the Colon of Immunologically Naive Swine" (2003). Biological Sciences Faculty Publications. 177.

https://digitalcommons.odu.edu/biology_fac_pubs/177

\section{Original Publication Citation}

Mansfield, L. S., Gauthier, D. T., Abner, S. R., Jones, K. M., Wilder, S. R., \& Urban, J. F. (2003). Enhancement of disease and pathology by synergy of Trichuris suis and Campylobacter jejuni in the colon of immunologically naive swine. American Journal of Tropical Medicine and Hygiene, 68(3), 70-80. 
Authors

Linda S. Mansfield, David T. Gauthier, Sheila R. Abner, Kathryn M. Jones, Stacey R. Wilder, and Joseph F. Urban 


\title{
ENHANCEMENT OF DISEASE AND PATHOLOGY BY SYNERGY OF TRICHURIS SUIS AND CAMPYLOBACTER JEJUNI IN THE COLON OF IMMUNOLOGICALLY NAIVE SWINE
}

\author{
LINDA S. MANSFIELD, DAVID T. GAUTHIER, SHEILA R. ABNER, KATHRYN M. JONES, STACEY R. WILDER, AND \\ JOSEPH F. URBAN \\ Department of Microbiology and Molecular Genetics, Michigan State University, East Lansing, Michigan; Nutrient Requirements and \\ Function Laboratory, Beltsville Human Nutrition Research Center, Agricultural Research Service, United States Department of \\ Agriculture, Beltsville, Maryland
}

\begin{abstract}
Campylobacter jejuni, a leading cause of bacterial gastroenteritis, has different age distribution and disease expression in developing and developed countries, which may be due to the endemnicity of infection and the age of acquisition of immunity. Differences in disease expression are not solely dependent on the $C$. jejuni strain or virulence attributes. Another modulating factor in developing countries may be endemic nematode infections such as Trichuris, which drive type 2 cytokine responses and down-regulate type 1 immune responses. In this study, three-day-old germfree pigs given dual infections with Trichuris suis and $C$. jejuni had more frequent, more severe diarrhea and severe pathology than pigs given no pathogens, only $T$. suis, or only $C$. jejuni. These pigs had significant hemorrhage and inflammatory cell infiltrates in the proximal colon where adult worms were found, and abscessed lymphoglandular complexes in the distal colon with intracellular $C$. jejuni. Pigs given only $C$. jejuni had mild clinical signs and pathology, and bacteria in feces or extracellular sites. Pigs given $T$. suis or no pathogens had no disease and minimal pathology. Thus, these agents synergized to produce significant disease and pathology, which was site specific.
\end{abstract}

\section{INTRODUCTION}

Disease in humans caused by Campylobacter spp. is a serious emerging problem in the United States ${ }^{1-3}$ and the world. ${ }^{4,5}$ Campylobacter jejuni is the most commonly reported bacterial cause of food-borne infection in the United States. ${ }^{6}$ In fact, $46 \%$ of laboratory-confirmed cases of bacterial gastroenteritis reported in the Centers for Disease Control and Prevention/U.S. Department of Agriculture/Food and Drug Administration Collaborating Sites Food Borne Disease Active Surveillance Network were caused by Campylobacter species. $^{6}$

Campylobacter jejuni causes a spectrum of disease in humans. Infection is initiated in the gastrointestinal tract, but can become extraintestinal in severe cases, particularly in immunocompromised hosts. ${ }^{7,8}$ In clinical reports describing primary infections with $C$. jejuni in developed countries, infection with mucosal disease predominates with symptoms of diarrhea, abdominal pain, and blood in the stool. ${ }^{9-11}$ However, infrequently, infection with systemic spread, infection without disease with short-term bacterial persistence, and infection with resistance and no bacterial persistence occur. ${ }^{9-11}$ In developing countries, $C$. jejuni enteritis usually occurs in infants; adults rarely have symptomatic disease. ${ }^{12}$ In these cases, the disease spectrum includes severe inflammatory illness, mild secretory diarrhea, or an asymptomatic carrier state. In addition, the duration of disease is shorter in children in hyperendemic areas compared with those with little previous exposure.

Campylobacter species possess significant genotypic and phenotypic diversity. ${ }^{13}$ However, C. jejuni strain variation does not account for all of these differences; the same strains producing watery diarrhea in children in developing countries have been isolated from visitors with acute inflammatory disease. ${ }^{14}$ In another study, there was no difference in serotypes isolated from symptomatic and asymptomatic children. ${ }^{15}$ The main explanation for the different disease expression in developing versus developed countries has been the early acquisition of immunity by children exposed to hyperendemic and/or polymicrobial infection. ${ }^{16-18}$ There is documentation of concurrent infections precipitating $C$. jejuni enteritis. ${ }^{19}$ These observations support the notion that this organism is capable of producing a spectrum of disease scenarios depending on the immune status of the host, virulence determinants of the bacterium, and complexity of concurrent infections.

In previous studies, inoculation of weaned swine with low levels of Trichuris suis (whipworm) initiated an interaction with resident bacterial flora to induce mucohemorrhagic enteritis in the colon. ${ }^{20}$ These pigs exhibited diarrhea, mucosal edema, inflammatory cell infiltration, and bacterial accumulation at the site of worm attachment in the proximal colon. In pigs infected with $T$. suis, lymphoglandular complexes (LGCs) in the submucosa of the distal colon were enlarged and contained numerous extra cellular and intracellular bacteria, eosinophils, lymphocytes, and macrophages. Control pigs infected with $T$. suis and treated with antibiotics had lesions localized to the site of worm attachment and histologically normal LGCs with no invasive bacteria in the distal colon. Another control group not inoculated with T. suis, but exposed to the same bacterial flora had no disease and no pathology. There was a $T$. suis dose-dependent increase in size of the follicular germinal centers and in the size of the lymphoid follicles within the LGCs. Multiple bacterial species were isolated from the LGC follicles; however, $C$. jejuni was the most common isolate. These results suggested that $T$. suis facilitated $C$. jejuni invasion of deeper tissues of the colon.

One billion people are infected with intestinal nematodes worldwide, and several million of these individuals are symptomatic. ${ }^{21,22}$ Ascaris lumbricoides, hookworms, and Trichuris trichiura are the most commonly diagnosed gastrointestinal nematodes associated with intestinal disease. ${ }^{22}$ Polyparasitism with hookworm, Ascaris, and Trichuris is common. ${ }^{23}$ It is possible that polymicrobial infections may explain some of the differences in clinical symptoms observed in young children with Campylobacter infections in developing countries. Based on the results in pigs, the following study was designed to examine whether a combined inoculum of $C$. jejuni and $T$. suis could synergize to enhance invasion of the colon by $C$. 
jejuni in an immunologically naive, germ-free pig and, if so, to explore the location of the invasion site. The results suggest that co-infection with a helminth that inhabits the proximal colon causes more severe consequences in immunologically naive pigs and could serve as a model to explore host resistance to $C$. jejuni, especially germinal center development in lymphoid tissues of the distal colon.

\section{MATERIALS AND METHODS}

Animals. All pigs were obtained by caesarian section at full term from two Landrace Yorkshire cross sows. The maintenance and care of experimental animals complied with the National Institutes of Health guidelines for the humane use of laboratory animals.

Preparation of germ-free incubators. Germ-free incubators were prepared in an animal containment facility. This facility had limited access with shower-in and shower-out facilities. Air entering the separate containment rooms was filtered and waste leaving the rooms was autoclaved or heated before disposal. Gnotobiotic swine incubators were sequentially disinfected and sterilized with Nolvasan (Fort Dodge Animal Health, Fort Dodge, IA), PolyClean (Madeira Inc., Spring, TX), and peracetic acid. All canned food and equipment required were passed through a barrier port and sterilized with peracetic acid spray.

Surgical procedure. Surgery was conducted in the germfree incubators. Piglets were taken from sows by caesarean section at approximately 112 days gestation under standard surgical procedure in the containment facility surgery room. Sows were given atropine $(0.045 \mathrm{mg} / \mathrm{kg})$ intramuscularly, sedated with Acepromazine ${ }^{\circledR}$ maleate (Boehringer Ingelheim Vetmedica, Inc., St. Joseph, MO) $(0.5 \mathrm{mg} / \mathrm{kg}$ intramuscularly), and an epidural anesthesia with $2 \%$ lidocaine was administered. Each sow was placed in lateral recumbency, and a standard surgical approach was performed through a paramedian incision. Pigs were delivered into sterile drapes and passed through a sterile sleeve into the gnotobiotic incubators by random assignment. When all piglets were delivered, the sow was given an overdose of sodium pentobarbital. Piglets were housed in germ-free incubators in individual cages in groups of 4-6 for social support and fed a sterilized complete milk diet (Esbilac; Borden Co., Hamshire, IL) for the duration of the experiment.

Experimental design. At three days of age, gnotobiotic pigs were infected with the following pathogens according to group; Group 1 (five pigs): control group (no infection), Group 2 (four pigs): infected with only with T. suis, Group 3 (five pigs): infected only with $C$. jejuni, and Group 4 (six pigs): infected with $T$. suis and $C$. jejuni. Pigs were examined three times per day for adverse signs. Abnormal findings, including descriptions and degree of diarrhea, were recorded. Fecal swabs were taken for bacteriologic isolation and identification on days 6 and 27 after infection. Pigs were maintained until severe clinical signs were observed in some, at which time, they were killed. At 27 days after infection, pigs were removed from the incubators and humanely killed with an intravenous overdose of sodium pentobarbital. Complete post mortem examinations were done, and tissues were recovered for various analyses.

Experimental infections. Trichuris suis. Embryonated eggs were prepared from adult whipworms isolated from colonic mucosa of experimentally infected pigs as described previously. ${ }^{24}$ Briefly, the worms were washed in sterile saline, then in sterile Hanks' balanced salt solution (sHBSS), incubated in $5 \times$ concentrated antibiotics $(500 \mathrm{U} / \mathrm{ml}$ of penicillin, $500 \mu \mathrm{g} / \mathrm{ml}$ of streptomycin, $1.25 \mu \mathrm{g} / \mathrm{ml}$ of amphotericin B, and $350 \mu \mathrm{g} / \mathrm{ml}$ of chloramphenicol) in RPMI 1640 medium for 16-24 hours, incubated in $1 \times$ antibiotic cocktail without chloramphenicol for 16-24 hours, and washed three times (two hours per wash) in sHBSS to remove residual antibiotics. Finally, worms were incubated for 10 days in RPMI 1640 medium containing 1\% glucose $\left(4\right.$ worms $/ \mathrm{ml}$ ) at $37^{\circ} \mathrm{C}$ in a humidified atmosphere of $5 \% \mathrm{CO}_{2}$ for collection of eggs. Eggs were allowed to embryonate, examined microscopically, and embryonated eggs were picked with a pipette into individual doses. Piglets in groups 2 and 4 were inoculated by oral gavage with 3,000 embryonated $T$. suis eggs.

Campylobacter jejuni. Pigs in groups 3 and 4 were inoculated orally with a low dose of $C$. jejuni $\left(1 \times 10^{6}\right.$ colonyforming units $[\mathrm{cfu}])$. The $C$. jejuni strain was second passage ATCC strain 33292 (American Type Culture Collection, Manassas, VA) isolated from a human with enteritis and characterized by testing with various media and conditions to optimize growth and ensure virulence. To test for virulence, three-day-old colostrum-deprived piglets were inoculated orally with $5 \times 10^{9} \mathrm{cfu}$ of $C$. jejuni strains 33291, 33292, and 33560. Piglets inoculated with strain 33292 developed diarrheal disease within two days post-inoculation and $C$. jejuni were re-isolated from feces, amplified to generate lowpassage bacterial stocks, and maintained in sheep blood at $-80^{\circ} \mathrm{C}$. Doses for infections were prepared from the second passage frozen stock. Bacteria were streaked onto Brucella agar supplemented with 5\% sheep blood and incubated for 48 hours at $37^{\circ} \mathrm{C}$ in an atmosphere of $5 \% \mathrm{CO}_{2}$. A single colony was picked into Brucella broth, which was grown at $37^{\circ} \mathrm{C}$ for 20 hours to early log phase. Confirmation of early log phase growth was based on growth curve determinations for strain 33292 , optical density readings at $560 \mathrm{~nm}\left(\mathrm{OD}_{560}\right)$, and dark field microscopy in which the majority of organisms showed a spiral form and darting motility. Doses were made by resuspending $0.5 \mathrm{~mL}$ of the $0.1 \mathrm{OD}_{560}$ inoculum in $49.5 \mathrm{~mL}$ of milk warmed to $37^{\circ} \mathrm{C}$. To confirm the desired inoculum of approximately $1 \times 10^{6} \mathrm{cfu}$ per pig, limiting dilution analyses was done on the prepared doses of $C$. jejuni.

Necropsy procedure. The entire gastrointestinal tract was removed, divided into small intestines, cecum, and large intestines, opened longitudinally, and walls were examined for pathologic lesions. A staging system developed previously was used to score and rank the severity of the colonic lesions. ${ }^{20}$ Twenty-five LGCs were measured from the colon of each pig using a micrometer to determine the overall expansion of the lymphoid follicles. After lesions were evaluated, tissue samples were taken, the remaining colon was scraped with a glass slide, and the scraped material was incubated with sterile saline $(0.85 \%)$ with $10 \mathrm{mM}$ EDTA for 30 minutes at $37^{\circ} \mathrm{C}$. After incubation, colonic scrapings were allowed to sediment for 30 minutes in graduated cylinders, the supernatant was aspirated, and the sediment was examined and enumerated for larval T. suis.

Histopathology. For each pig, full-thickness tissue samples were taken from the cecum, proximal colon, and distal colon for histopathologic evaluation. All samples were fixed in $10 \%$ formalin, embedded in paraffin, sectioned at $5 \mu \mathrm{m}$, stained 
MANSFIELD AND OTHERS

with hematoxylin and eosin, and sections were observed and photographed (Optiphot-2 microscope with an H-III automatic camera; Nikon, Corporation, Tokyo, Japan) A scoring system was developed to evaluate histopathologic changes in representative villus crypt units of the proximal and distal colon from each pig. Each feature was given a rank from 0 to 4 (e.g., $0=$ few cells or no pathologic change, $1=$ slight cellular infiltrate, mild pathologic change, $2-3=$ a moderate number of cells or moderate pathologic change, and $4=$ many cells or maximal pathologic change). Specific features that were evaluated were as follows. The lumen was evaluated for excess mucus and inflammatory exudate. The epithelium was evaluated for surface integrity, number of intraepithelial lymphocytes, goblet cell hypertrophy, goblet cell depletion, crypt hyperplasia, crypt atrophy, adenomatous change in the crypt, and crypt inflammation. The lamina propria and submucosa were evaluated for increases in inflammatory or immune cells and their distribution, and the submucosa was evaluated for fibrosis. The thickness of the lamina propria, submucosa, and muscularis from the proximal and distal colon was measured in five fields with an ocular micrometer and the average thickness for these layers was recorded for each pig.

Immunohistochemistry. Twenty-five LGCs, full-thickness sections of the proximal and distal colon, and colonic Peyer's patches from the cecum immediately distal to the ileal aperture were sampled in their entirety for immunohistochemical analysis. Tissue snips were overlayed with OCT compound (Sakura Finetek, Inc., Torrance, CA), snap frozen in liquid nitrogen, and maintained at $-80^{\circ} \mathrm{C}$ until use. Tissues were sectioned at 4-6 $\mu \mathrm{m}$ with a cryostat (Reichert-Jung Cryocut Bensheim, Germany). Sections were adhered to either polyL-lysine (Sigma, St. Louis, MO)-coated glass slides or charged glass slides (Fisher Scientific, King of Prussia, PA) and fixed in $4^{\circ} \mathrm{C}$ acetone for 5 minutes.

Tissue sections were ringed with PAP marker (Research Products International Corp., Mount Prospect, IL), rehydrated with TTBS (50 mM Tris, $0.1 \%$ Tween $20,150 \mathrm{mM}$ $\mathrm{NaCl}$ ) for $10 \mathrm{~min}$, and cross-reactive sites were blocked with B-TTBS (TTBS plus 1\% bovine serum albumin) for $30 \mathrm{~min}$. The primary monoclonal antibody (anti-C. jejuni outer membrane neat hybridoma supernatants or 1:500 purified ascites; Biogenesis, Sandown, NH) was diluted 1:10 with B-TTBS. Other sections from the LGCs were prepared and stained with the monoclonal antibody 5C9, which is directed against the IgM- $\mu$ b of swine (kindly provided by Dr. Joan Lunney, U.S. Department of Agriculture, Beltsville, MD). Negative controls were treated with B-TTBS only instead of primary antibody, and a mixture of irrelevant anti-rat monoclonal antibodies. Sections were incubated with primary antibody at $22^{\circ} \mathrm{C}$ for three hours, washed with TTBS, and incubated for one hour with biotin-labeled $F(a b ')_{2}$ fragments of sheep anti-mouse antibody (Sigma) diluted 1:100 in B-TTBS (previously cross-absorbed against human serum). Sections were then incubated for one hour with streptavidin-alkaline phosphatase (Sigma, St. Louis, MO) diluted 1:200 in TBS (50 $\mathrm{mM}$ Tris, $150 \mathrm{mM} \mathrm{NaCl}$ ), followed by three washes (five minutes per wash) with TTBS. For colorimetric detection, $5 \mathrm{mg}$ of naphthol AS-MX phosphate (Sigma) was dissolved in $250 \mathrm{~mL}$ of N,N-dimethylformamide and added to $10 \mathrm{mg}$ of Fast-Red TR salt (Sigma) in TTBS. This solution was applied to tissue sections for 10-15 minutes, or until the background stain increased. Sections were counterstained with Gill's I hematoxylin for 15 seconds and rinsed for two minutes in distilled water. The slides were dried and coverslips applied using aqueous mounting medium (Biomeda Corp., Foster City, CA).

Electron microscopy. Another set of samples from the same tissues was placed into Timm's fixative for electron microscopy to correlate ultrastructural findings to individual cell data and to identify Campylobacter bacteria in situ. ${ }^{25}$ All samples were fixed for transmission electron microscopy (TEM) by immersing the pellet in 20 volumes of osmium tetroxide for one hour with gentle agitation. The samples were washed three times (10 minutes per wash) in Sorenson's buffer, followed by three five-minute washes in sterile, double-distilled water. Samples were then incubated in $0.5 \%$ uranyl acetate overnight, washed in sterile, double-distilled water, and dehydrated in a graded ethanol series. Following dehydration, the samples were infiltrated by passing through increasing concentrations of Spurr's medium (3:1, 1:1, 1:3, $100 \%$ ethanol to Spurr's medium) ${ }^{25}$ Specimens were embedded in Spurr's medium, cut in 60-90-nm sections on a Sorvall microtome (Kendro Laboratory Products, Asheville, NC), mounted on HX 200 copper grids, examined using a $100 \mathrm{CX}$ electron microscope (JEOL, Inc., Peabody, MA), and photographed.

Statistical analysis. The size and number of the LGCs, the number of $T$. suis, and the number of episodes of a particular clinical sign from the four treatment groups were compared using the Kruskal-Wallis rank sum test. The pathology scores were expressed as the percentage of pigs with a particular stage of pathology over the total number of pigs evaluated.

\section{RESULTS}

Dose determinations. Campylobacter jejuni. Brucella broth cultures of $C$. jejuni harvested after 20 hours of incubation had an OD value of 0.114 , and approached $100 \%$ spiral shapes and darting motility. The results of limiting dilution analyses of three replicate doses were $5.3 \times 10^{6}, 4.2 \times 10^{6}$, and $4.0 \times 10^{6}$ (mean dose $\left.=4.5 \times 10^{6}\right)$, which ensured uniformity of the doses given to pigs in groups 3 and 4 .

Trichuris suis. Pigs in groups 2 and 4 were given 3,000 T. suis eggs in $5 \mathrm{~mL}$ of diluent. The actual percentage of the $T$. suis dose recovered was $0.1-1 \%$ (mean $\pm \mathrm{SD}=17 \pm 13$ ) in Group 2 ( $T$. suis only) and $0.3-1 \%($ mean \pm SD $=21 \pm 11)$ in Group 4 (T. suis and C. jejuni). Although the doses were low, all $T$. suis-infected pigs had $T$. suis and uninfected groups had no $T$. suis.

Clinical signs. Clinical signs experienced by all pigs are summarized in Table 1. Diarrhea was the most prominent adverse sign. Uninfected pigs had 11 episodes of diarrhea. Pigs given only $T$. suis had nine episodes of diarrhea. Pigs given both $T$. suis and $C$. jejuni had 68 episodes of severe diarrhea with blood and inflammatory cells. Pigs given only $C$. jejuni had transient fever for 24 hours after infection. They had 11 episodes of diarrhea over the observation period, which did not correlate temporally with fever. One pig each in groups 1,3 , and 4 died at day one of age before the experimental infections were performed due to blood loss when the umbilical clamps came off. Campylobacter jejuni was isolated at 6 and 27 days post-infection from feces of all pigs in groups 3 and 4 , but not from any pig in groups 1 and 2 . 
TABLE 1

Clinical signs of disease in gnotobiotic swine from day 1 through day 27 post-infection with Trichuris suis and Campylobacter jejuni

\begin{tabular}{|c|c|c|c|c|}
\hline $\begin{array}{l}\text { Clinical } \\
\text { signs }\end{array}$ & $\begin{array}{l}\text { Group 1 } \\
\text { uninfected } \\
\left(\mathrm{n}=4^{*}\right)\end{array}$ & $\begin{array}{l}\text { Group } 2 \\
\text { T. suis } \\
(\mathrm{n}=4)\end{array}$ & $\begin{array}{l}\text { Group } 3 \\
\text { C. jejuni } \\
\left(\mathrm{n}=4^{*}\right)\end{array}$ & $\begin{array}{l}\text { Group 4 } \\
\text { T. suis and C. jejuni } \\
\left(\mathrm{n}=5^{*}\right)\end{array}$ \\
\hline Fever & $1 \dagger$ & 0 & 6 & 6 \\
\hline Depression & 4 & 4 & 5 & 3 \\
\hline Poor appetite & 0 & 2 & 1 & 2 \\
\hline Dehydration & 3 & 3 & 1 & 0 \\
\hline Diarrhea & 11 & 9 & 11 & $68 \ddagger$ \\
\hline
\end{tabular}

Pathology. At 27 days after infection, severe pathology was present only in the colon of pigs that had both $T$. suis and $C$. jejuni infections (Table 2 and Figure 1D). Four of five pigs in this group had mucosal hemorrhage over more than $50 \%$ of the colon surface. The colon was inflamed, thickened, edematous, the surface epithelium was denuded in some areas, and many LGCs were filled with mucopurulent debris. Uninfected pigs and pigs with only $T$. suis had little gross pathology (Table 2 and Figure 1A and C). Two pigs given only C. jejuni had slight hyperemia located mainly in the Peyer's patches of the small intestines and in small areas of the proximal colon surrounding and including the ileocecal Peyer's patches (Table 2 and Figure 1B). In pigs infected with both T. suis and C. jejuni, there was a statistically significant increase in the size and in the number of LGCs in the distal colon $(P \leq 0.05)$ (Table 3).

Histopathology. The presence of histopathologic lesions in the colon of pigs depended on infection status (Figures 2 and 3). Uninfected control pigs had no lesions and served as the control for evaluating increases in thickness of the layers of the proximal colon (Figure 2, Group 1 and Figure 3A). Their mean \pm SD values were mucosal $=295 \pm 5 \mu \mathrm{m}$, submucosal $=57 \pm 16 \mu \mathrm{m}$, and muscularis $=110 \pm 23 \mu \mathrm{m}$. Pigs given only $T$. suis had crypt hyperplasia and increased thickness of all layers of the proximal colon (mucosal $=346 \pm 24$ $\mu \mathrm{m}$, submucosal $=73 \pm 21 \mu \mathrm{m}$, and muscularis $=142 \pm 2$ $\mu \mathrm{m}$ ) with goblet cell hypertrophy (Figure 2, Group 2 and Figure $3 C$ ). Pigs given only $C$. jejuni had mild lesions and no crypt hyperplasia $($ mucosal $=298 \pm 4 \mu \mathrm{m}$, submucosal $=72$

TABLE 2

Colon pathology scores of experimental pigs 27 days after infection

\begin{tabular}{|c|c|c|c|c|}
\hline Pathology* & $\begin{array}{l}\text { Group } 1 \\
\text { uninfected }\end{array}$ & $\begin{array}{c}\text { Group } 2 \\
\text { Trichuris suis }\end{array}$ & $\begin{array}{c}\text { Group } 3 \\
\text { Campylobacter jejuni }\end{array}$ & $\begin{array}{c}\text { Group } 4 \\
\text { T. suis and C. jejuni }\end{array}$ \\
\hline Stage 1 & $5 / 5$ & $4 / 4$ & $3 / 5$ & $0 / 5$ \\
\hline Stage 2 & $0 / 5$ & $0 / 4$ & $2 / 5$ & $1 / 5$ \\
\hline Stage 3 & $0 / 5$ & $0 / 4$ & $0 / 5$ & $3 / 5$ \\
\hline Stage 4 & $0 / 5$ & $0 / 4$ & $0 / 5$ & $1 / 5$ \\
\hline
\end{tabular}

* Stage 1 = healthy colon unaffected by parasites or bacteria, gut is smooth and tan, $0.50-1.50 \mathrm{~mm}$ in thickness, and lymphoglandular complexes (LGCs) contain follicles made up of lymphocytes and macrophages with normal entrapped mucosal glandular crypts; Stage $2=$ colon has patchy areas of hemorrhage $(<50 \%$ of the colon is hemorrhagic), is $1.51-2.50$ $\mathrm{mm}$ in thickness, is slightly roughened, and LGCs have mild to moderate infiltrates of inflammatory cells, including eosinophils; Stage $3=$ colon is hemorrhagic $(>50 \%$ of it surface), $2.50 \mathrm{~mm}$ or greater in thickness, is markedly roughened, and LGCs appear with mucopurulent debris within the entrapped glandular crypts; Stage $4=$ colon is covered with a fibrinonecrotic pseudomembrane, is $2.50 \mathrm{~mm}$ or greater in thickness, may or may not have denuded areas where the mucosa is sloughed, and LGCs, if not obliterated by pseudomembranes, are filled with mucopurulent debris. Scores greater than 1 were considered abnormal pathology. $\pm 2 \mu \mathrm{m}$, and muscularis $=197 \pm 50 \mu \mathrm{m}$ ) or goblet cell hypertrophy (Figure 2, Group 3 and Figure 3B). Pigs with dual infections had the greatest crypt hyperplasia with increased thickness of all layers of the colon (mucosal $=324 \pm 9 \mu \mathrm{m}$, submucosal $=96 \pm 2 \mu \mathrm{m}$, and muscularis $=160 \pm 39 \mu \mathrm{m})$ and goblet cell hypertrophy (Figure 2, Group 4 and Figure 3D).

Cellularity was increased in the proximal colon of pigs given $T$. suis, $C$. jejuni, or both pathogens compared with those that were uninfected (Figures 2 and 3). More marked infiltration of cells was seen in the pigs with dual infections in both the proximal and distal colon (Figure 3D). These pigs had large numbers of lymphocytes, macrophages, neutrophils, plasma cells, and eosinophils in the lamina propria and the submucosa (Figure 2, Group 4). Only one of five pigs given only $C$. jejuni had all of these cell types (Figure 2, Group 3 and Figure $3 \mathrm{~B}$ ). The predominant reactive cell types in pigs given only $T$. suis were lymphocytes, macrophages and eosinophils, which were in higher numbers than in the pigs infected only with $C$. jejuni (Figure 2, Group 2 and Figure 3C).

Immunohistochemistry. IgM staining of the LGCs showed that germinal centers were present in all pigs given either $T$. suis, $C$. jejuni, or both. All pigs given $C$. jejuni had germinal centers in the LGCs, but the overall follicle size was significantly increased only in pigs infected with both agents (Table 3 and Figure 4D).

The distribution of $C$. jejuni in the mucosal epithelium and the LGCs was evaluated using a monoclonal $\mathrm{IgG}_{1}$ antibody directed against the cell surface of the bacterium (Figure 5). Pigs with only $C$. jejuni had anti-C. jejuni staining in the LGCentrapped crypts and within the follicle-associated epithelium of the LGCs (four of four pigs infected with $C$. jejuni) (Figure 5C). Pigs infected with both $T$. suis and $C$. jejuni had anti- $C$. jejuni staining in the LGC-entrapped crypts, within the follicle-associated epithelium of the LGCs, and within individual cells of the lymphoid follicle (five of five pigs infected with $C$. jejuni and T. suis) (Figure 5D). In the dual infected group, there was overall more anti-C. jejuni staining because the follicles were significantly larger than in the group given only C. jejuni. In the distal colon, none of the LGCs that were sectioned had $T$. suis adults or larvae within the nodule. Histology confirmed the presence of LGCs in all uninoculated, untreated, control pigs and in those pigs infected only with $T$. suis, but they were small, had no apparent germinal centers, and no anti-C. jejuni staining (none of two pigs infected with T. suis and none of four control pigs) (Figure 5A and B).

Electron microscopy. Electron microscopy was used to examine the colonic mucosa and LGCs from pigs in groups 3 and 4 (Figures 6 and 7). In the mucosal samples of pigs infected only with $C$. jejuni, there were lumenal bacteria, epithelial cell-associated bacteria, goblet cell-associated bacteria, and crypt-associated bacteria, but no bacteria within epithelial cells, the lamina propria, or beneath the muscularis mucosae (Figure 6). Dually infected pigs had bacteria in the lumen, associated with epithelial cells, associated with goblet cells, associated with the crypts, and within some epithelial cells (Figure 7). One pig in this group had $C$. jejuni in moderate numbers in the lamina propria and beneath the muscularis mucosae.

The LGCs from both groups had follicle-associated epithelia lining the entrapped crypts of Lieberkuhn. These epithelia had specialized cells with $\mathrm{M}$ cell characteristics, which were among epithelial cells between the lumen of the crypt and the 


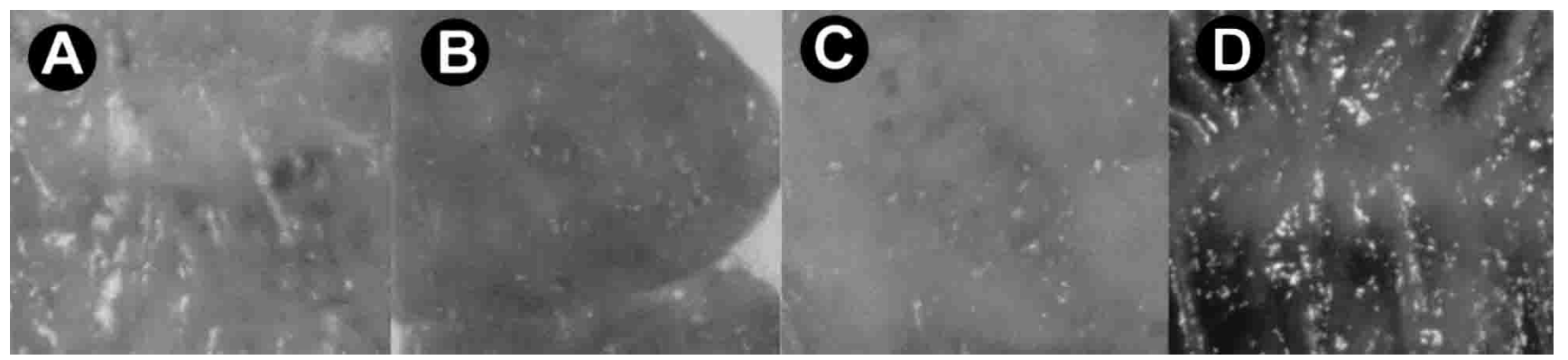

Figure 1. Lesions (gross pathology) in the proximal colon of representative pigs of different infection status. A, an uninfected pig. B, a pig infected only with Campylobacter jejuni. $\mathbf{C}$, a pig infected only with Trichuris suis. D, a pig infected with both $T$. suis and $C$. jejuni.

lymphoid follicle. The $\mathrm{M}$ cells were connected to adjacent cells by tight junctions and had invaginations in the basolateral membrane containing lymphocytes and macrophages. They had apical microvilli, which were thicker, and more irregular than flanking enteroabsorptive cells. In pigs given only $C$. jejuni, no intracellular $C$. jejuni bacteria were observed (Figure 6). In LGCs, bacteria were found in entrapped crypt mucus and superficially associated with goblet cells of the follicle-associated epithelium (Figure 6a-d). Pigs with dual infections had LGCs invaded by $C$. jejuni. Bacteria were found in epithelial cells in the follicle-associated epithelium, as well as in crypt mucus and goblet cells (Figure $7 \mathrm{a}-\mathrm{d}$ ). In these pigs, some $C$. jejuni were within vacuoles while others had no limiting phagocytic membrane that could be detected around bacteria within the epithelial cells.

\section{DISCUSSION}

These results demonstrate that $C$. jejuni and $T$. suis synergize in the colon of immunologically naive, germ-free pigs to produce disease and pathologic lesions. The clinical signs and lesions are similar to those observed in 6-8-week-old conventionally reared pigs with mucohemorrhagic colitis from which we isolated $C$. jejuni. ${ }^{20}$ In the present study, pigs with both pathogens had diarrhea with blood and mucus containing leukocytes similar to that observed in susceptible humans and primates with $C$. jejuni enteritis. ${ }^{8,26}$ Diarrhea correlated temporally with the presence of third and fourth larval stages of $T$. suis in crypts of the proximal colon, although only low numbers of the parasite were recovered from these pigs. These low numbers were likely due to low viability of the embryonated eggs or to the inherent difficulty in recovering larval stages from the intestines. The numbers recovered are probably an underestimate of the actual level of infection

TABLE 3

Size and total number of lymphoglandular complexes in the colon of experimental pigs 27 days after infection with either Trichuris suis, Campylobacter jejuni, both pathogens, or neither pathogen*

\begin{tabular}{lcc}
\hline \multicolumn{1}{c}{ Treatment group } & Number $\dagger$ & Size $(\mathrm{mm}) \dagger$ \\
\hline Group 1, Uninfected & $325 \pm 125 \ddagger$ & $0.75 \pm 0.28 \ddagger$ \\
Group 2, T. suis & $266 \pm 153 \ddagger$ & $0.80 \pm 0.57 \ddagger$ \\
Group 3, C. jejuni & $620 \pm 377 \ddagger$ & $1.30 \pm 0.67 \ddagger$ \\
Group 4, T. suis and C. jejuni & $860 \pm 313 \S$ & $2.40 \pm 0.40 \S$ \\
\hline * Values are the mean \pm SD. & \\
$\quad \begin{array}{l}\dagger \\
\text { Wallis rank with different superscripts were significantly different }(P \leq 0.05) \text { by Kruskal- }\end{array}$
\end{tabular}

because they are small and difficult to visualize at this stage. Low numbers of parasites may explain why pigs given only $T$. suis had no clinical signs and minimal pathologic lesions. Pigs given only $C$. jejuni had transient fever and, thereafter, no clinical signs, but shed the organism in feces throughout the 27-day course of the experiment, suggesting that their gastrointestinal tracts were colonized.

In susceptible humans, $C$. jejuni may colonize either the small $^{27,28}$ or large intestines, but most often results in colitis or typhlitis. ${ }^{29}$ Invasion of cells is suggested by the presence of abdominal pain, fever, diarrhea, frank blood in stools, and inflammatory cells in stools. ${ }^{10,29}$ Histologic sections of colonic biopsies show that patients with acute colitis have crypt abscesses, depletion of goblet cells, and inflammatory infiltrates of the lamina propria composed of polymorphonuclear leukocytes, lymphocytes, and plasma cells. ${ }^{29,30}$ Monocytes may play a role in the translocation of the organism to the blood stream, but the role of phagocytosis as a defense mechanism against $C$. jejuni seems to be secondary with the humoral response having the primary role against infection. ${ }^{8}$

There was site specificity of the pathologic lesions observed in dual infected pigs 27 days after infection. In the proximal colon where the worms reside, there was hemorrhage, crypt hyperplasia, thickening of submucosa and muscularis layers, edema, epithelial cell destruction, goblet cell hypertrophy and increased numbers of lymphocytes, macrophages, neutrophils, and eosinophils in the lamina propria and in the submucosa. In the distal colon, LGCs were significantly enlarged due to crypt abscesses and enlarged germinal centers. Pigs infected with $T$. suis only had mild crypt hyperplasia and goblet cell hypertrophy in the proximal colon and normal LGCs, suggesting that bacteria such as $C$. jejuni are required to produce the degree of pathology usually observed in field cases. Only two of four pigs given only $C$. jejuni had pathology with mild hyperemia in Peyer's patches of the small intestines and colon. Possibly lymphoid tissues serve as a reservoir in swine for $C$. jejuni to maintain the colonized state.

Campylobacter jejuni were isolated and observed in the colon of all pigs receiving the bacterium. However, the presence of $T$. suis greatly influenced the location of $C$. jejuni. Pigs with dual infections had $C$. jejuni in crypt abscesses in the mucosa and the LGCs, associated with the surface of goblet cells, within epithelial cells especially in the follicle-associated epithelium of the LGCs, and within the lamina propria in inflamed areas of the proximal colon. Pigs infected only with 


\section{A. Proximal Colon}

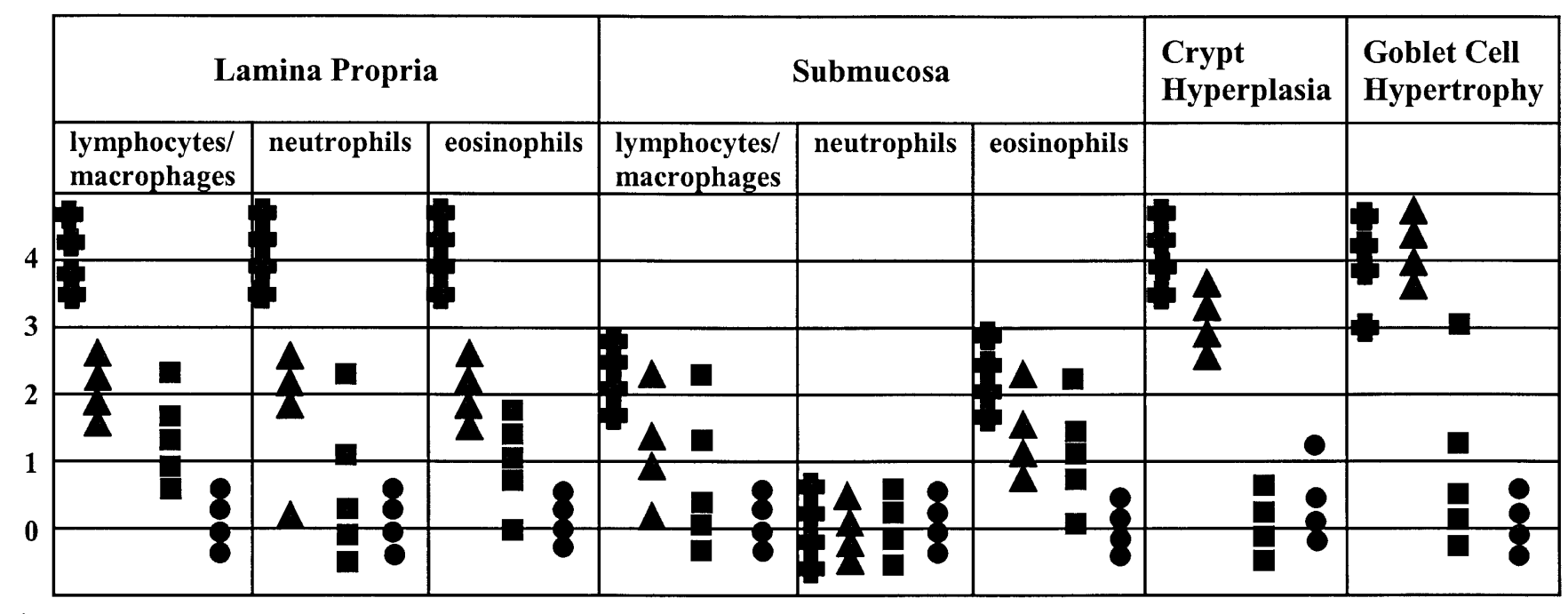

\section{B. Distal Colon}

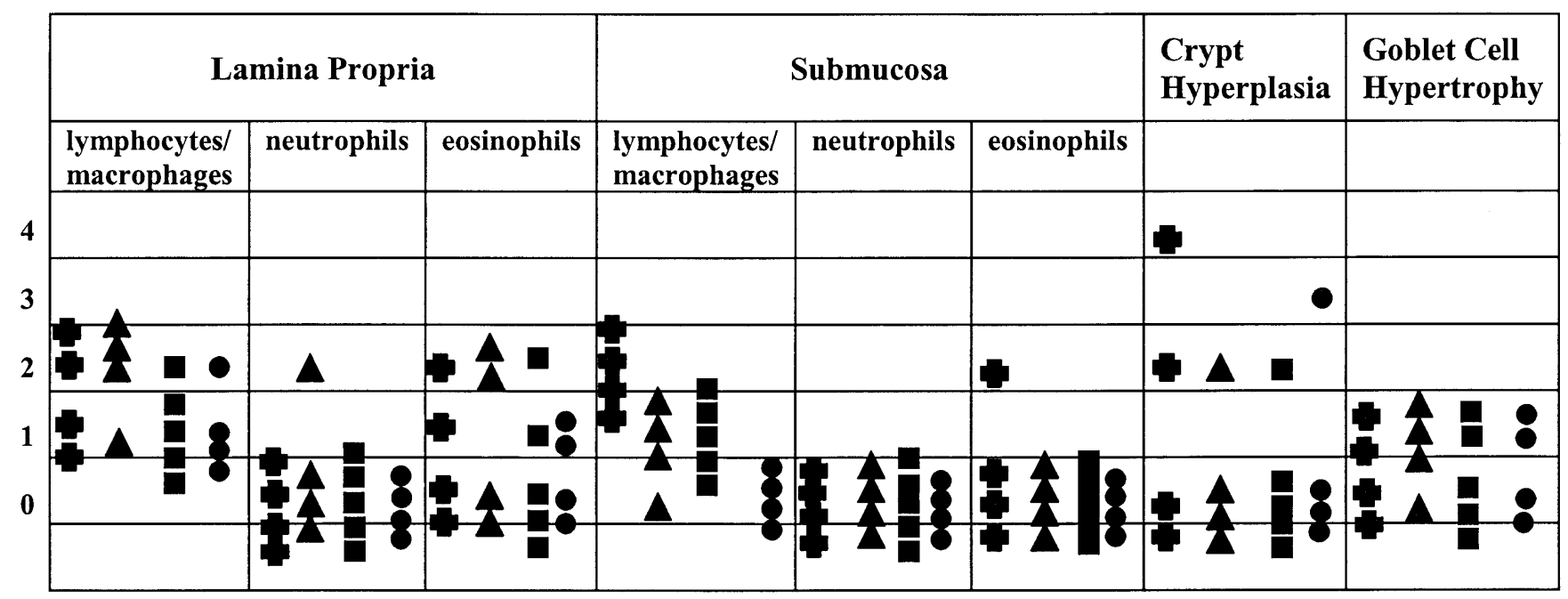

FIGURE 2. Effect of infection with Trichuris suis, Campylobacter jejuni, both pathogens, or neither pathogen on the histopathologic changes in the proximal (A) and distal (B) colon of gnotobiotic pigs. Circles represent Group 1 (uninfected pigs), triangles represent Group 2 (pigs infected only with $T$. suis), squares represent Group 3 (pigs infected only with $C$. jejuni), and crosses represent Group 4 (pigs infected with both $T$. suis and $C$. jejuni). Ranking scores ranging from 0 (none) to 4 (many) are given for each pig in each category (column) depending on the number of each cell type, or the degree of crypt hyprerplasia or hypertrophy. The number of symbols in each column indicates the number of pigs with that score.

C. jejuni had C. jejuni in colonic sections, especially in colonic crypts, where they were found mainly in the lumen or associated with mucus near goblet cells, but not within epithelial cells.

Babakhani and others found similar pathology when they orally infected newborn, colostrum-deprived pigs with a different invasive strain of $C$. jejuni. ${ }^{31}$ On necropsy of pigs from day 1 to day 6 after infection, they observed edema, hyperemia and mucus, as we saw in our dually infected pigs at day 27. In their study, histopathologic examination and transmission electron microscopy demonstrated damage to surface epithelial cells and the presence of intracellular bacteria in the large intestines. In our studies, it is unlikely that the group infected only with $C$. jejuni experienced pathology as severe as observed early in infection in their study because clinical signs were absent, except for transient fever. These varying results appear to be largely due to the different $C$. jejuni doses of these two virulent strains that were used. However, differences in C. jejuni strain virulence, the absence of other enteric flora, or genetic differences in the swine hosts may also play a role. In our laboratory, preliminary infections of germ-free pigs with a high dose of $C$. jejuni strain 33292 produced acute disease similar to that seen by Babakhani and others. ${ }^{31}$ Also, in several studies, dual infected pigs with naturally acquired C. jejuni showed the most significant effects 21-27 days after infection with $T$. suis. These data suggest that the worm has 


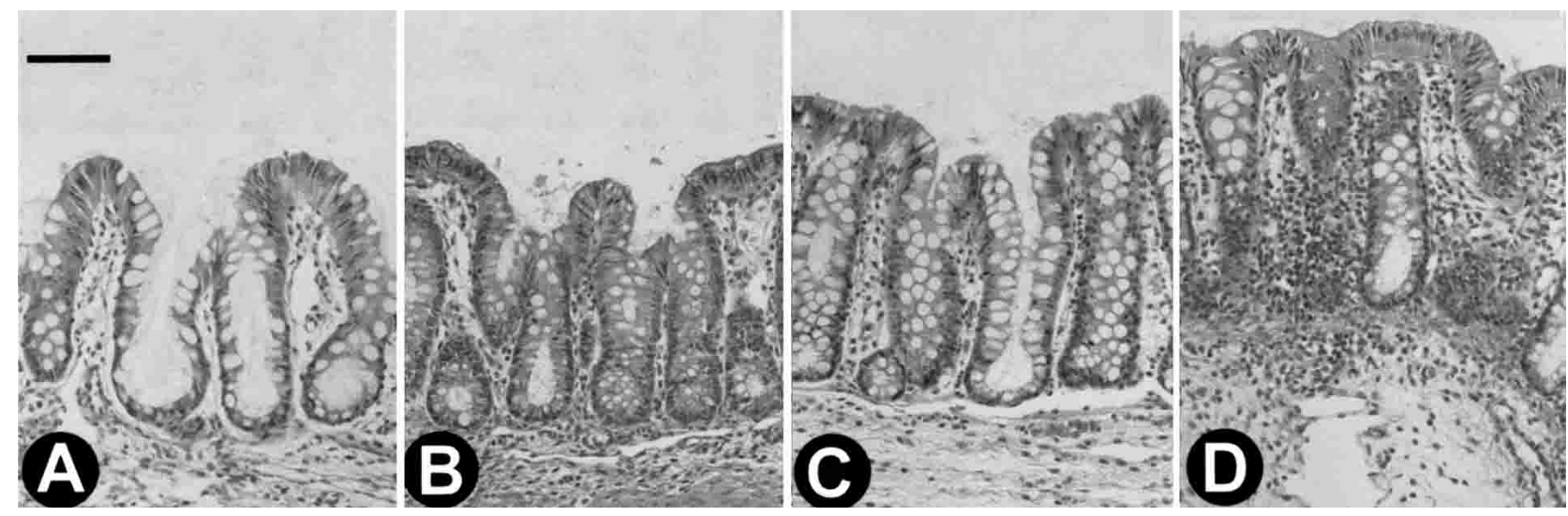

FIGURE 3. Representative hematoxylin and eosin-stained sections of the proximal colon of gnotobiotic pigs of different infection status. Each section shows the mucosa and the submucosa aligned at the base of the submucosa, but excludes the muscularis. Bars represents $20 \mu \mathrm{m}$. A, an uninfected pig; B, a pig infected only with Campylobacter jejuni; $\mathbf{C}$, a pig infected only with Trichuris suis; $\mathbf{D}$, a pig infected with both T. suis and C. jejuni. Note the increased thickness in $\mathbf{C}$ and $\mathbf{D}$ compared with $\mathbf{A}$ and $\mathbf{B}$, and the marked cellular infiltrate in $\mathbf{D}$.

an effect and bacterial strain differences alone do not account for the differences in timing of the expression of pathology in these studies. Despite the differences in the experimental systems, the cell invasion pattern and electron microscopic and histopathologic lesions observed in our study and that of Babakhani and others ${ }^{31}$ were similar. Recent data shows that $C$. jejuni has an adhesin that binds to a cellular receptor. ${ }^{32}$ Ketley has hypothesized two models to explain $C$. jejuni epithelial cell invasion. ${ }^{11} \mathrm{He}$ suggests motility and binding to epithelial cells via an adhesin leads to invasion by either 1) endocytic uptake via coated pits and association of the vacuole with microtubules, or 2) interaction with molecules associated with caveolae leads to transduction of a signal resulting in endocytosis involving actin filaments. Our data does not conclusively support either mechanism and suggests that a time course infection in young pigs with labeled $C$. jejuni with and without $T$. suis would be useful.

The LGCs appear to be important sites of $C$. jejuni colonization/invasion and immune induction sites for $C$. jejuni. This is likely due to their characteristic anatomy. They are submucosal secondary lymphoid structures in the distal colon of swine, humans, cattle, dogs, and other animals. ${ }^{33}$ They have structural features similar to Peyer's patches with propria nodules that lie beneath the muscularis mucosae and mucosal glands that pass through the muscularis mucosae and lie among the nodule. A specialized follicle-associated epithelium (FAE) is present in these entrapped glands. The FAE directly overlays the follicle and forms the lining of entrapped, radially ramifying crypts within the follicle. It is similar to the FAE of jejunal and ileocecal Peyer's patches, being composed of goblet cells, enteroendocrine cells, columnar and cuboidal enterocytes, and M-type cells associated with intra-epithelial lymphocytes. ${ }^{34}$ In this study, C. jejuni invaded the entrapped crypts of the LGCs, and all pigs exposed to $C$. jejuni had enlarged germinal centers in the LGCs. These findings suggest that $C$. jejuni is processed by $\mathrm{M}$ cells in the LGC FAE even in the absence of $T$. suis. In dually infected pigs, the dramatic increase in the size of the LGCs, expansion of the germinal centers, and the presence of $C$. jejuni within epithelial cells of the FAE suggest that $T$. suis enhances this response. Epithelial cells appear to be damaged in $C$. jejuni infections in most people with primary infections $;^{10}$ however, the mechanisms controlling this are not known. In these pigs, electron microscopy showed that $C$. jejuni does invade epithelial cells in the FAE of the LGC. Also, immunohistochemistry showed anti-C. jejuni staining of cells with reticular morphology within the LGC follicles of dual infected pigs. It may be that macrophages within the LGC propria nodule, and especially in the pocket of $\mathrm{M}$ cells underlying the LGC FAE, are important in translocation of the bacterium from the lu-
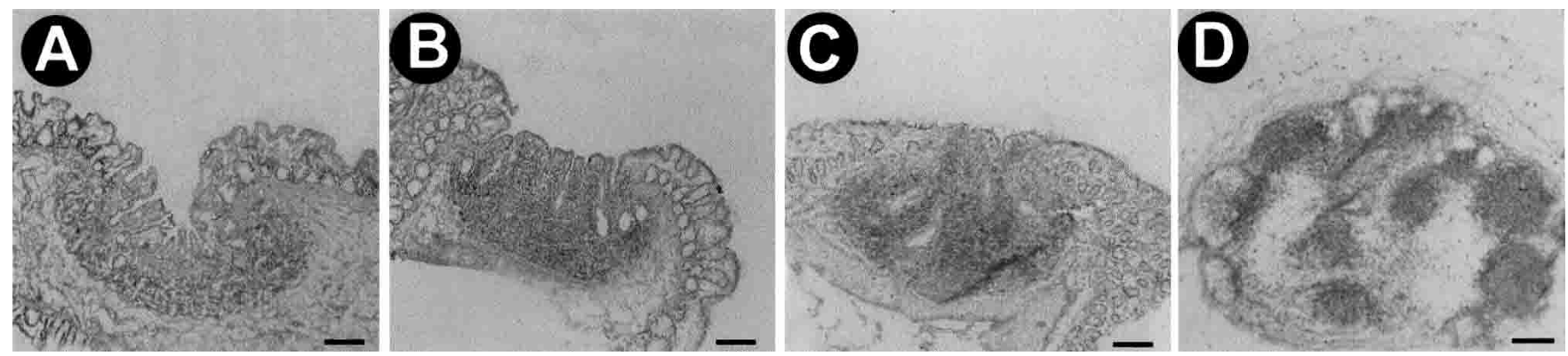

FIgURE 4. IgM immunohistochemical staining (A, B, and $\mathbf{C}$ have red stain with blue counterstain and $\mathbf{D}$ has blue stain with no counterstain) of representative lymphoglandular complexes (LGCs) from the distal colon of gnotobiotic pigs of different infection status. The magnification is the same in all panels. Bars represent $100 \mu \mathrm{m}$. The LGCs are shown from $\mathbf{A}$, an uninfected pig; B, a pig infected only with Trichuris suis; $\mathbf{C}$, a pig infected only with Campylobacter jejuni; D, a pig infected with both T. suis and C. jejuni. Note the increased size of the LGC from a dual-infected pig, as well as the increased size of the germinal centers of the lymphoid follicles. 

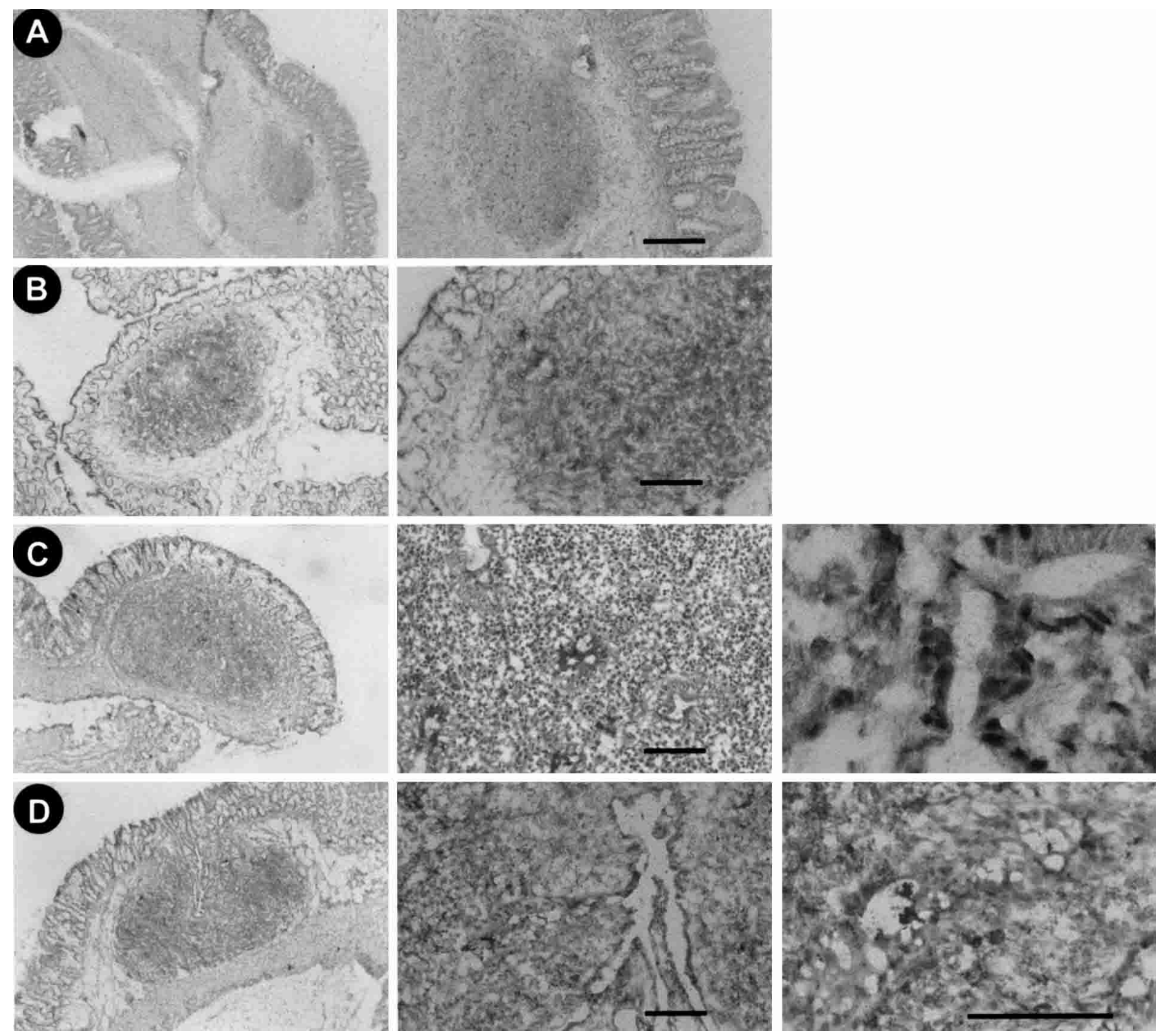

FIGURE 5. Immunohistochemical staining (red stain) using a monoclonal antibody for Campylobacter jejuni of lymphoglandular complexes (LGCs) from the distal colon of gnotobiotic pigs of different infection status. The magnification increases from left to right in all panels. Bars represent $20 \mu \mathrm{m}$. Images show an LGC from $\mathbf{A}$, an uninfected pig; $\mathbf{B}$, a pig infected only with Trichuris suis; $\mathbf{C}$, a pig infected only with Campylobacter jejuni; $\mathbf{D}$, a pig infected with both T. suis and C. jejuni. Panels in $\mathbf{C}$ show $C$. jejuni-stained cells mainly in the follicle-associated epithelium. Panels in D show C. jejuni-stained cells in the follicle-associated epithelium and cells with reticular morphology within the LGC follicle.

men. Campylobacter jejuni has been found in $\mathrm{M}$ cells in Peyer's patches of rabbits. ${ }^{35}$ This structure may provide a route for dissemination to deeper tissues and possibly the blood stream. Further studies with labeled $C$. jejuni are needed to identify the cells with $C$. jejuni inside the LGC follicle. Pigs given only $C$. jejuni had smaller LGCs with smaller germinal centers. The majority of $C$. jejuni-specific immunohistochemical staining of these LGCs was limited to the FAE of the entrapped crypt, but electron microscopy showed no bacteria within these cells. Therefore, we believe that anti-C. jejuni staining of epithelial cells in pigs infected only with $C$. jejuni is due to binding of antibody to $C$. jejuni breakdown products within vacuoles. In previous experiments, the propria nodules of LGCs increased in size with increasing numbers of $T$. suis inocula. ${ }^{20}$ Therefore, it was surprising that in this experiment pigs given only $T$. suis had no significant increase in the size of germinal centers in the LGCs. It appeared that the increase in size of the germinal centers was in response to the presence of $C$. jejuni. The nature of this epithelium, its interface with the lymphoid follicle, and the results of these experiments supports the role of the LGC in antigen sampling.

The mechanism by which $T$. suis synergize with $C$. jejuni is unknown but is likely to involve direct mechanical damage as well as cytokine-mediated damage. It is known that in the proximal colon $T$. suis first stage larvae invade basal crypt cells where they develop in a syncytium of epithelial cells. ${ }^{36}$ In this study, it appears that the majority of lesions are due to bacteria, but the parasite is required to elicit disease and pathology. It is likely that the lesions seen in dual-infected pigs resulted from the compound assault of colonic crypts by $T$. suis and $C$. jejuni. Earlier studies supported a synergistic relationship of $T$. suis and the microbial flora in producing the 

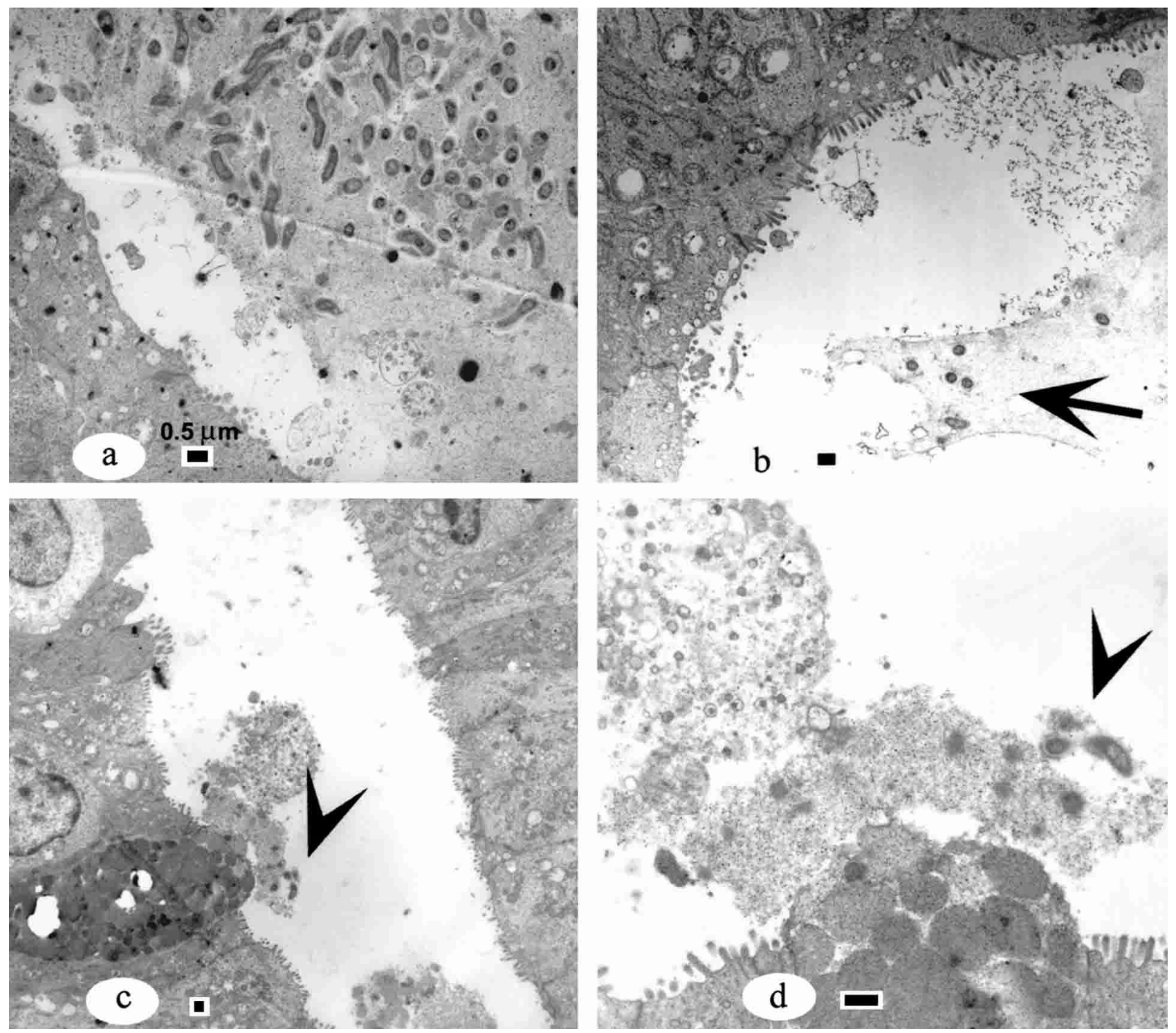

FIGURE 6. Electron micrograph of the follicle-associated epithelium of a lymphoglandular complex of a gnotobiotic pig 27 days after infection with only Campylobacter jejuni. Bars represent $0.5 \mu \mathrm{m}$. a, Follicle-associated epithelium of a pig showing campylobacter jejuni in the mucus in the crypt lumen. b, Follicle-associated epithelium of another infected pig showing bacteria in mucus of the crypt lumen (arrow). No bacteria were observed within epithelial cells in a or b. c, Goblet cells in follicle-associated epithelium (arrowhead). d, Higher magnification view of $C$. jejuni associated with a goblet cell (arrowhead).

pathogenesis of mucohemorrhagic colitis in swine. ${ }^{37}$ Trichuris suis excretory-secretory products (ESPs) affect epithelial cell integrity, ${ }^{38}$ and ESPs cause increases in numbers of $C$. jejuni within in vitro cultured cells if cells are treated before addition of C. jejuni (L. D. Cunningham, Mansfield L. S., unpublished data). We speculate that $T$. suis larvae in the proximal colon may expose extracellular matrix necessary as cellular targets for adhesins of the bacterium such as fibronectin. ${ }^{32}$

Cytokine dysregulation may be an important contributor to this phenomenon, but functions differently than in inbred mice with similar dual infections. Trichuris causes marked inflammation and strong TH-2-associated immune responses in the colon of young experimentally infected, conventionally reared pigs, ${ }^{20,39}$ which apparently leads to susceptibility to $C$. jejuni. This model has proved useful for converting a $C$. jejuni colonized state to a disease state to examine the role of immune modulation in the intestine as a contributor to the patho- genesis of Campylobacter infections. Additionally, hyper-Th-1 polarized interleukin (IL)-10/4 knockout (KO) mice infected with $T$. muris developed severe ulcerating lesions, which were reversed by antibiotic treatment. ${ }^{40}$ However, it will be important to examine the difference between these systems and that of concommitant Helicobacter felis and Heligomoisoides polygyrus in C57BL/6 mice in which the helminth down-regulates immune-mediated pathology in the stomach due to the bacterium. ${ }^{41}$ Likewise, Toxoplasma gondii and Schistosoma mansoni synergize to promote hepatocyte dysfunction in C57BL/6 mice, but gut pathology is decreased in dual infected mice. ${ }^{42}$ Differences in worm species, worm location, worm-feeding mechanisms, and the stereotypical immune response patterning of these inbred mice compared with outbred pigs and specific IL10/4 KO mice could explain the differences observed.

Received May 18, 2002. Accepted for publication September 11, 2002. 

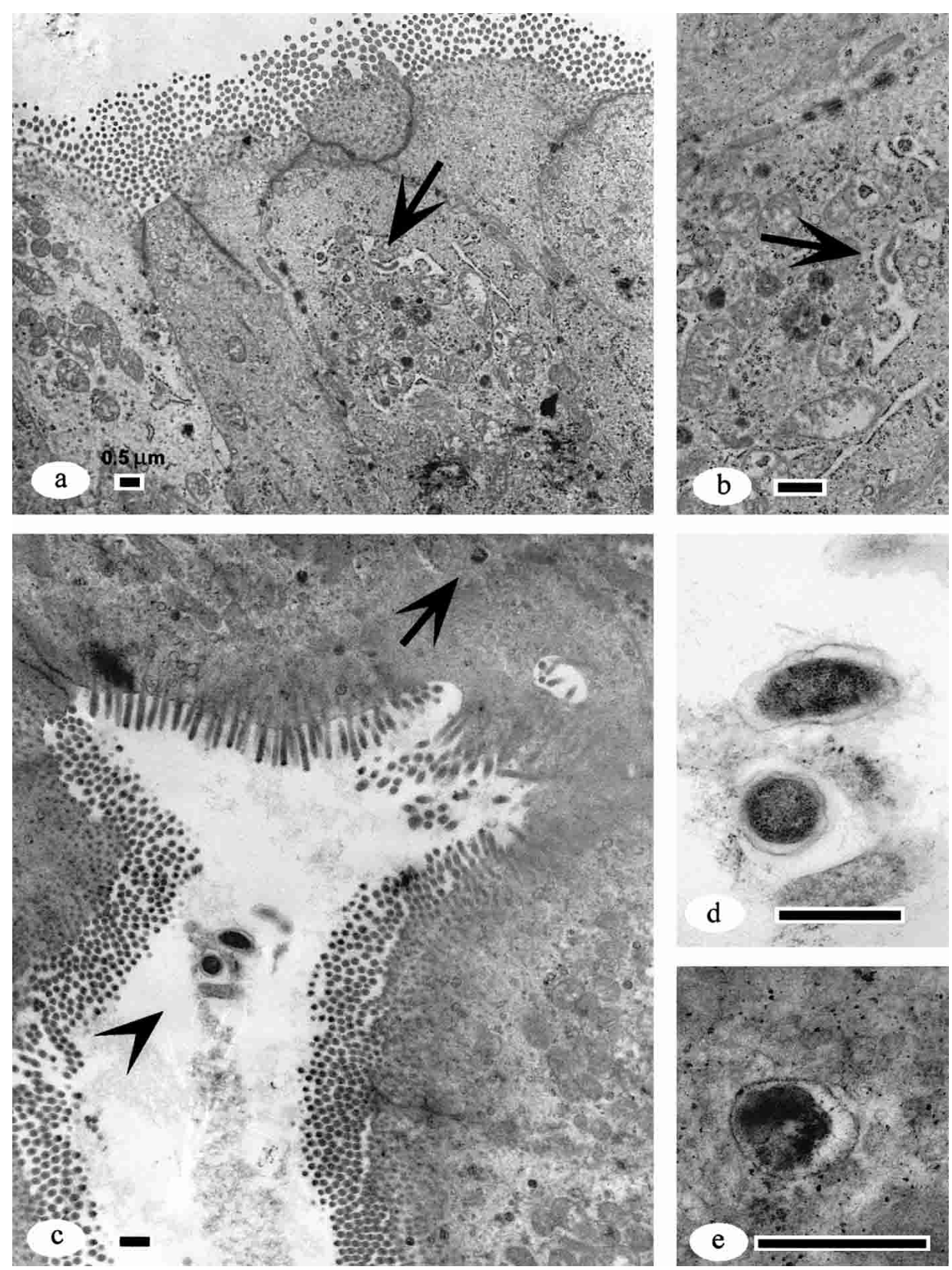

FIGURE 7. Electron micrograph of the follicle-associated epithelium of a lymphoglandular complex of a gnotobiotic pig 27 days after infection with Campylobacter jejuni and Trichuris suis. Bars represent $0.5 \mu \mathrm{m}$. a, Follicle-associated epithelium showing $C$. jejuni within epithelial cells (arrow). b. Higher magnification view of a. c, Epithelial cells in the follicle-associated epithelium of the lymphoglandular complex. Note the bacteria in mucus in the entrapped crypt (arrowhead). The arrow indicates an epithelial cell. d, Higher magnification view of $C$. jejuni in the lumen of the crypt (arrowhead in c). e, Higher magnification view of $C$. jejuni within an epithelial cell (arrow in $\mathbf{c})$.

Acknowledgments: We thank Dr. Robert Holland for providing training with anesthesia and surgical techniques for the caesarian sections of the donor sows, Dr. Jon Patterson for reviewing the histopathologic and electron microscopic images, Sally Burns for technical assistance with electron microscopy, and Dr. Joan Lunney for providing monoclonal antibodies. We also thank Awdry Jones for expert technical assistance in providing $T$. suis eggs for infection of pigs.

Financial support: This work was supported by National Institutes of Health grant 61-0954, U.S. Department of Agriculture Animal Health Formula Funds grant 6881, National Food Safety and Toxicology Center grants 71-2954 and 71-9487, Michigan State University (East Lansing, MI), and Agricultural Research Service grant CHRIS 1265-32000-049.
Authors' addresses: Linda S. Mansfield, David T. Gauthier, Sheila R. Abner, Kathryn M. Jones, and Stacey R. Wilder, B43 Food Safety Toxicology Center, Department of Microbiology and Molecular Genetics, Michigan State University, East Lansing, MI 48824, Telephone: 517-432-6309, Fax: 517-432-2310, E-mail: Mansfie4@cvm. msu.edu. Joseph F. Urban, Nutrient Requirements and Function Laboratory, Beltsville Human Nutrition Research Center, Agricultural Research Service, United States Department of Agriculture, Beltsville, MD 20705.

\section{REFERENCES}

1. CDC, 1997. Food borne diseases active surveillance network, 1996. MMWR Morb Mortal Wkly Rep 46: 258-261. 
2. FSIS, 1995. Food Safety Research: Current Activities and Future Needs. Washington, DC: USDA.

3. Buzby JC, Roberts T, 1996. ERS updates US food borne disease costs for seven pathogens. Food Rev 19: 20-25.

4. World Health Organization, 1995. Progress towards health for all: third monitoring report. World Health Stat Q 48: 190.

5. Gore A, 1996. Emerging infections threaten national and global security. ASM News 62: 448-449.

6. Altekruse SF, Stern NJ, Fields PI, Swerdlow DL, 1999. Campylobacter jejuni - an emerging food borne pathogen. Emerg Infect Dis 5: 28-35.

7. Sorvillo FJ, Lieb LE, Waterman SH, 1991. Incidence of campylobacteriosis among patients with AIDS in Los Angeles County. J Acquir Immune Defic Syndr Hum Retrovirol 4: 598-602.

8. Wallis MR, 1994. The pathogenesis of C. jejuni. Br J Biomed Sci 51: 57-64.

9. Karmali MA, Fleming PC, 1979. Campylobacter enteritis in children. J Pediatr 94: 527-533.

10. Black RE, Levine MM, Clements ML, Hughes TP, Blaser MJ, 1988. Experimental Campylobacter jejuni infection in humans. J Infect Dis 157: 472-479.

11. Ketley JM, 1997. Pathogenesis of enteric infection by Campylobacter jejuni. Microbiology 143: 5-21.

12. Blaser MJ, Glass RI, Hug MI, Stoll B, Kibrya GM, Alim ARMA, 1980. Isolation of Campylobacter fetus subsp. jejuni from Bangladeshi children. J Clin Microbiol 12: 744-747.

13. Guerry P, Szymanski CM, Prendergast MM, Hickey TE, Ewing CP, Pattarini DL, Moran AP, 2002. Phase variation of Campylobacter jejuni 81-176 lipooligosaccharide affects ganglioside mimicry and invasiveness in vitro. Infect Immun 70: 787-793.

14. Skirrow MB, Blaser MJ, 1992. Clinical and epidemiological considerations. Nachamkin I, Blaser MJ, Tompkins LS, eds. Campylobacter jejuni: Current Status and Future Trends. Washington, DC: American Society for Microbiology, 3-8.

15. Sjogren E, Ruiz-Palacios G, Kaijser B, 1989. Campylobacter jejuni isolations from Mexican and Swedish patients, with repeated symptomatic and/or asymptomatic diarrhoea episodes. Epidemiol Infect 102: 47-57.

16. Taylor DN, Echeverria P, Pitarangsi C, Seriwatana J, Bodhidatta L, Blaser M, 1988. Influence of strain characteristics and immunity on the epidemiology of Campylobacter infections in Thailand. J Clin Microbiol 26: 863-868.

17. Calva JJ, Ruiz-Palacios GM, Lopez-Vidal AB, Ramos A, Bojalil $\mathrm{R}, 1988$. Cohort study of intestinal infection with Campylobacter in Mexican children. Lancet $i: 503-506$.

18. Richardson NJ, Koornhoff HJ, Bockenheuser VD, 1981. Longterm infections with Campylobacter fetus subsp. jejuni. J Clin Microbiol 13: 846-849.

19. Konkel ME, Joens LA, 1990. Effect of enteroviruses on adherence to and invasion of HEp-2 cells by Campylobacter isolates. Infect Immun 58: 1101-1105.

20. Mansfield LS, Urban JF, 1996. The pathogenesis of necrotic proliferative colitis in swine is linked to whipworm-induced suppression of mucosal immunity to resident bacteria. Vet Immunol Immunopathol 50: 1-17.

21. World Health Organization, 1981. Intestinal protozoan and helminthic infections. World Health Organ Tech Rep Ser 666: $1-152$.

22. Bundy DA, 1994. Immunoepidemiology of intestinal helminthic infections. 1. The global burden of intestinal nematode disease. Trans $R$ Soc Trop Med Hyg 88: 259-261.

23. Bundy DA, Cooper ES, 1989. Trichuris and trichuriasis in humans. Adv Parasitol 28: 107.
24. Hill DE, Gamble HR, Rhoads ML, Fetterer RH, Urban JF, 1993. Trichuris suis: a zinc metalloprotease from culture fluids of adult parasites. Exp Parasitol 77: 170-178.

25. Barta JR, Boulard Y, Desser SS, 1987. Ultrastructural observations on secondary merogony and gametogony of Dactylosoma ranarum Labbe 1894 (Eucoccidiida; Apicomplexa) J Parasitol. 73: 1019-1029.

26. Russell RG, O'Donnoghue M, Blake DC, Zulty J, DeTolla LJ, 1993. Early colonic damage and invasion of Campylobacter jejuni in experimentally challenged infant Macaca mulatta. $J$ Infect Dis 168: 210-215.

27. King EO, 1957. Human infection with Vibrio foetus and a closely related Vibrio (letter). J Infect Dis 101: 119.

28. Cadranel S, Rodesch P, Butzler JP, Dekeyser P, 1973. Enteritis due to 'related Vibrio' in children. Am J Dis Child 126: 152155.

29. Lambert ME, Schofield PF, Ironside AG, Mandal BK, 1979. Campylobacter colitis. BMJ 1: 857-859.

30. Mandal BK, DeMol P, Butzler JP, 1984. Campylobacter Infection in Man and Animals. Boca Raton, FL: CRC Press, 21-31.

31. Babakhani FK, Bradley GA, Joens LA, 1993. Newborn piglet model for campylobacteriosis. Infect Immun 61: 3466-3475.

32. Konkel ME, Garvis SG, Tipton SL, Anderson DE Jr, Cieplak W Jr, 1997. Identification and molecular cloning of a gene encoding a fibronectin-binding protein $(\mathrm{CadF})$ from Campylobacter jejuni. Mol Microbiol 24: 953-963.

33. Mansfield LS, Hill DE, Urban JF, 1995. Lymphoglandular complexes process antigen in the distal colon in swine. Keusch GT, Kawakami M, eds. Cytokines, Cholera and the Gut. Amsterdam: IOS Press, 185-195.

34. Morfitt DC, Pohlenz JF, 1989. The porcine colonic LGC complex: distribution, structure and epithelium. Am J Anat 184: 41-51.

35. Walker RI, Schmauder-Chock EA, Parker JL, 1988. Selective association and transport of $C$. jejuni through $\mathrm{M}$ cells of rabbit Peyer's patches. Can J Microbiol 34: 1142-1147.

36. Beer RJS, 1973. Morphological descriptions of the egg and larval stages of Trichuris suis Schrank, 1788. Parasitology 67: 263278.

37. Rutter JM, Beer RJS, 1975. Synergism between Trichuris suis and the microbial flora of the large intestine causing dysentery in pigs. Infect Immun 11: 395-404.

38. Abner SR, Hill DE, Turner JR, Black ED, Bartlett P, Urban JF, Mansfield LS, 2002. Response of intestinal epithelial cells to Trichuris suis excretory-secretory products and the influence on Campylobacter jejuni invasion under in vitro conditions. $J$ Parasitol 88: 738-745.

39. Urban JF, Madden KB, Svetic A, Cheever A, Trotta PP, Gause WC, Katona IM, Finkelman FD, 1992. The importance of Th2 cytokines in protective immunity to nematodes. Immunol Rev 127: 205-220.

40. Schopf LR, Hoffman KF, Cheever AW, Urban JF Jr, Wynn TA, 2002. IL-10 is critical for resistance and survival during gastrointestinal helminth infection. J Immunol 168: 2383-2392.

41. Fox JG, Beck P, Dangler CA, Whary MT, Wang TC, Shi HN, Nagler-Anderson C, 2002. Concurrent helminth infection modulates inflammation and gastric immune responses and reduces Helicobacter-induced gastric atrophy. Nat Med 6: 536542.

42. Marshall AJ, Brunet LR, van Gessel Y, Alcaraz A, Bliss SK, Pearce EJ, Denkers EY, 1999. Toxoplasma gondii and Schistosoma mansoni synergize to promote hepatocyte dysfunction associated with high levels of plasma TNF- $\alpha$ and early death in C57BL/6 mice. J Immunol 163: 2089-2097. 